\title{
Where is the human waist? Definitions, manual compared to scanner measurements ${ }^{1}$
}

\author{
Daisy Veitch ${ }^{\mathrm{a}, *}$ \\ ${ }^{a}$ SHARP Dummies Pty Ltd, 102 Gloucester Avenue, Belair, 5052, South Australia, Australia
}

\begin{abstract}
Where exactly is the human waist? How do definitions work for women who deviate from the conventional body shape? Does the measuring instrument matter?

Waist is conventionally understood to be a measurable zone within the abdominal region of the torso, a zone of considerable importance. There needs to be a good consistent waist definition, one accurate and valid for everyone. Incorrect definition and measurement will result in technical errors, commercial wastage and customer dissatisfaction. This paper investigates the waist's location and size from the point of view of garment construction for 90 adult women scanned and manually measured in a breast reduction study at Flinders Medical Center, South Australia.
\end{abstract}

There are differing definitions of the location of the human waist as well as different measuring instruments. This study compares:

- Two definitions:

- $\quad$ ISO 8559, 2.1.11 and

- CAESAR, Waist Circumference Preferred.

- Two different instruments:

- the traditional tape measure, and

- $\quad$ software-extracted computer-aided anthropometry (CAA)

Substantial discrepancies between the results from these two locations-definitions were found. The choice of instrument used seriously affects the measurement obtained. This study demonstrates three things:

- waist is not horizontal for a significant sub group of the population,

- CAA extracted waist measurements are not accurate (same as real values) or valid (measures the characteristic) for a sub group, and

- manually measured CAESAR Preferred Waist accurately and validly measured all individuals studied.

There is a clear need to modify ISO waist definition for garment construction to include the full range of anatomical variation encountered amongst women.

Keywords: ISO 8559, waist, body scanning, computer-aided anthropometry, garment construction

\section{Introduction}

Waist is a commonly identified anatomical region of the human body. All cultures have a notion of waist, no doubt in part due to the great practical significance of carrying objects attached to the human body and of supporting clothing. Belts, sashes and waistbands are amongst the most common articles of clothing. Recently, measurement of waist circumference has become an important clinical tool in studies of obesity. [10]

\footnotetext{
${ }^{1}$ This paper is based on the data collected during the Breast Reduction study by AFESA (Anthropometry for Elective Surgery Assessment), whose other members are: Maciej Henneberg, Phil Griffin, Nicola Dean, Karen Burford, Kalavani Zeitouneh, Phillipa Van Essen and others. We are grateful to the AFESA Team for the opportunity to use these data. Thanks also to Martin McAvoy for editorial assistance, Griselda Raisa Susanto for data entry, analysis and secretarial support and David Summerhayes for preparation of the images.

*Corresponding author. E-mail: daisy@sharpdummies.com.au
} 
Most human beings can identify where their waist is, and yet there is no universal, repeatable and comparable way to measure waist circumference precisely. This situation is caused partly by large anatomical variation in human body shapes, and partly by the differing meanings of 'waist' in different applications.

So what is the human waist, and where exactly? How does the end use of the measurement affect its definition? How do two waist definitions, the ISO (International Organization for Standardization) 8559:1989 and CAESAR (Civilian American and European Surface Anthropometry Resource Project-CAESAR ${ }^{\circledR}$ ) waist definitions work for women who show substantial difference from the idealized female torso shape? Lastly, are manual tape measurements and scan extracted waist measurements interchangeable? Does the measuring instrument matter?

Waist is generally understood to be situated in the abdominal region in the middle of the torso, and is a zone of significance for design such as clothing construction $[7,9,17]$, or as a measure of health $[5,12,14]$ etc. The waistline is defined as the line or contour of the waist. This paper investigates the waist location and size from the point of view of garment construction.

A number of formal waist definitions for apparel exist. These include:

1) "ISO 8559:1989 Garment construction and anthropometric surveys - Body dimensions [3]... 2.1.11, waist girth: The girth of the natural waistline between the top of the hip bones (iliac crests) and the lower ribs, measured with the subject breathing normally and standing upright with the abdomen relaxed (see figure 7).”

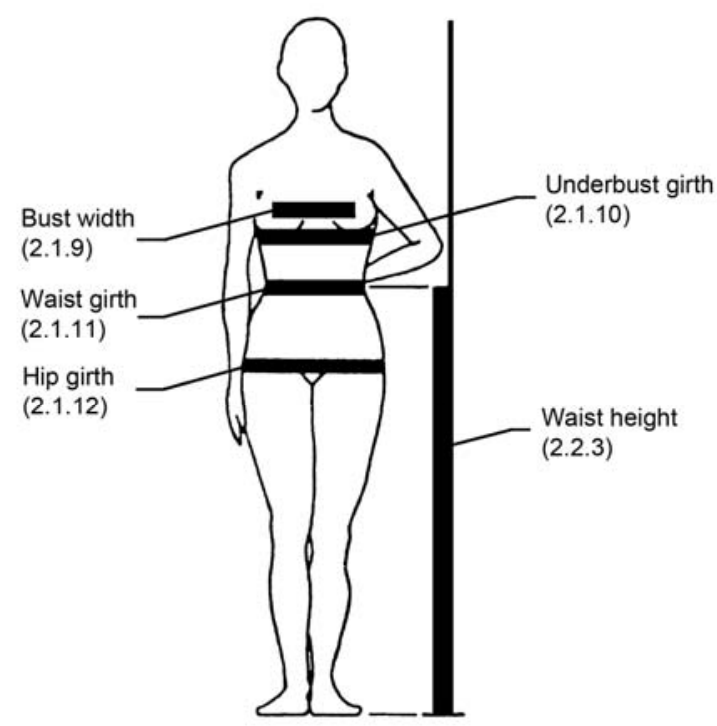

Figure 7

Fig. 1. ISO 8559 "Figure 7"

Author's note: The picture shows the measurement as horizontal in addition only one waist height is required, so although not specified, it is assumed to be horizontal. In addition, no measuring instrument is specified. No gender is specified although all the pictures show woman.

2) “37. CAESAR Name: WAIST CIRCUMFERENCE PREFERRED,

ISO Reference No. N/A,

ISO Name:

Description: Maximum circumference of the waist at the subject's 'preferred' waist level. Method: subject stands fully erect with the weight distributed equally on both feet and the arms hanging freely downwards. The subject's feet are placed in footprints adhered to the standing surface (the foot prints are positioned approximately $10 \mathrm{~cm}$ apart at the heels and rotated 33 degrees at the toes). The subject's preferred waist level is marked by using an elastic band.

NOTE: Preferred waist level is established by the subject, who places an elastic band at the level he or she would prefer to wear the waist of their pants.

Instrument: Steel tape measure.'[4]

3) “JIS Z 8500:2002 Ergonomics - Basic human body measurements for technological design. Waist circumference: horizontal circumference of trunk at a level midway between the lowest ribs and 
the upper iliac crest. This is the same with ISO 7250 1.

JIS L 0111:2006 Glossary of terms used in body measurements for clothes. Waist girth: circumference length of waistline. Waistline: where the waist belt is settled at midway between the lowest ribs and ilium. Not necessarily horizontal.'[13]

Author's note: This is very similar to ISO 8559 2.1.11; therefore it was not added as a separate measurement.

4) ASTM D5219-09 Standard Terminology Relating to Body Dimensions for Apparel Sizing

"waist girth, ... - the minimum horizontal circumference around the body at waist height."[1]

5) "Australian Standard AS1344-1997 Size coding scheme for women's clothing - Underwear, outerwear and foundation garments... In preparation of this Standard ISO $3635 \ldots$ ISO $3637 \ldots$ ISO $8559 \ldots$ and ISO $4416 \ldots$ were consulted."'[2]

Author's note: The Australian standard cross-references the ISO 8559; thus it is assumed in this paper to be the same measurement.

6) Other surveys use other definitions. For example, Chung et al. [6] used another definition of waist, i.e., horizontal circumference measured at the height of the navel, in measuring 7800 Taiwanese children aged 6-18 for a clothing sizing system. ISO 8559 definition was not used because "the location of the natural waistline may vary from person to person", but the navel was used as an "easy landmark to identify while taking the measurement"[6].

Further complications arise with the use of various tools for waist measurement and different measurement techniques. Since waist circumference is a surface measurement over soft tissues of the trunk, compressibility of these tissues may result in various measuring tools giving different results for the same subject depending on how much soft tissues were compressed by a measuring tape. Wider tapes will cause more compression than narrow tapes. For practical and ethical reasons, waist circumference may be measured over garments that compress soft tissues to various extents while the thickness of a garment's fabric is included in the measurement. Newly developed digital tools, like laser scanners, do not compress tissues at all, but they encounter a different set of problems related to difficulties extracting measurements from scans [16], and the inability to manually remove organs or tissues that in some individuals overhang the waist line through normal anatomical variation (see Figure 6). In the ISO, no measuring instrument is specified, whereas CAESAR states that the measurement must be taken with a steel tape.

In this paper we assess the accuracy and validity of different techniques of measuring waist circumference in two ways. First we compare measurements following definitions 1) and 2) above (validity). Then we compare measurements taken manually (tape measurements) and those extracted from scans (accuracy).

\section{Methods}

\subsection{Participant recruitment and data collection}

Approval was granted for this study from Flinders Clinical Research Ethics Committee (application \#269108). Participants were 90 adult women (aged from 18 to 72) enrolled in a breast reduction study at Flinders Medical Center, South Australia. They were measured both manually with a steel tape measure and also by CAA using Cyberware WBX scanner, Monterey, CA and CySize software, headus (metamorphosis), WA, Australia. [8]

Each participant had her waist measured manually by a criterion anthropometrist following the CAESAR Preferred Waist approach. Women were asked to indicate their preferred waist according to the CAESAR definition outlined above. Prior to taking any measurements scan markers were placed manually on the front, back and both sides of their preferred waistline to ensure measurement location accuracy and repeatability. Also the highest points of their iliac crests were marked. The waist circumference was manually measured by passing a steel measuring tape (Lufkin) over the four waist markers.

Each participant was then scanned by a Cyberware WBX whole body scanner in a standing position with arms adducted $30 \mathrm{~cm}$ from the hip.[4, 18] Each scan was individually calibrated using CyScan software. Preferred waist circumferences were extracted from scans using the same landmarks as those used for manual measurement. The second ISO defined waist circumference was extracted from the scan. This waist height was determined by using the back waist marker as waist height and then following the ISO defined waist, which is assumed horizontal. Both scan extracted measures use the software CySize [8], and used the $\mathrm{T}$ or tape measure function that spans indents and is designed to act like a physical tape measure. The CAA data were extracted by experienced operators. 
Data were subjected to statistical analyses using descriptive statistics, parametric tests of significance and correlation and regression analyses.

\section{Results}

For the CAESAR preferred waist definition, measurements obtained manually with a tape and those digitally extracted from scans were very similar and highly correlated (Figure 1). A bivariate graph shows the close relationship.

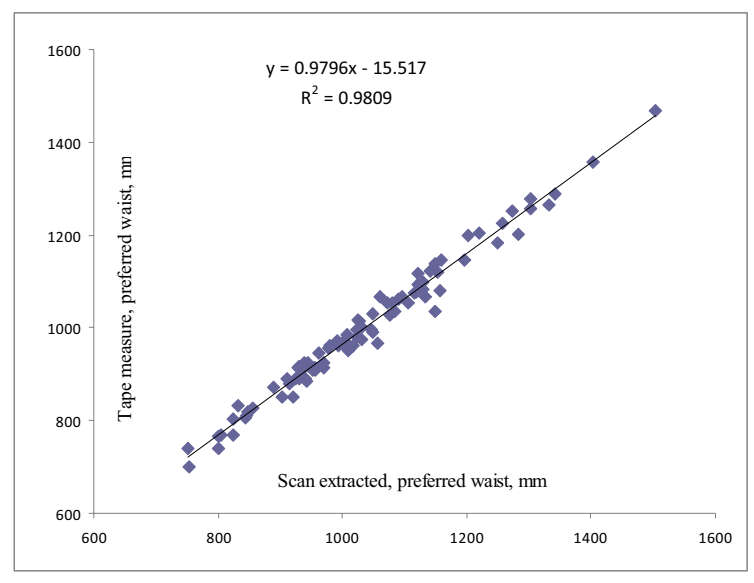

Fig. 1: Scanner Vs Manual Measurements (both CAESAR 37. Preferred Waist)

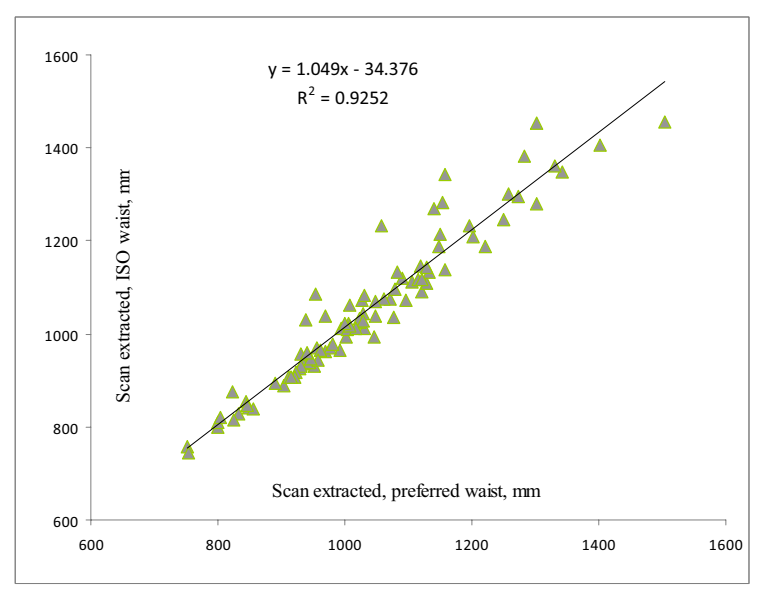

Fig. 2: Bivariate plot showing preferred waist Vs ISO 8559 waist (both scan derived)

Waist circumferences, however, obtained with the same digital measuring tool for different definitions, were excellent for some individuals but discordant for others; see Figure 2. In other words, these two definitions of circumferences require measurement in different locations on some individuals, leading to substantial discrepancies.

Investigation of various individuals reveal why. Figure 3 shows a normal individual who matches the idealized perception of a woman's body. The pink line marking her waist is the ISO defined waist and the yellow line underneath show CAESAR preferred waist. Both match and are horizontal.

All participants could be measured manually using the CAESAR definition of preferred waist with the steel tape. However not all could be measured digitally. In addition, there were a number of cases in which ISO waist was obviously measuring in a way that was not anticipated by the original ISO committee. See Figures 4, 5 and 6 . The magnitude of the errors is shown in Table 1.

Table 1 shows the percentage of participants in the study whose difference between CAESAR preferred waist and ISO waist was one clothing size or more, where one clothing size equals $50 \mathrm{~mm}$.

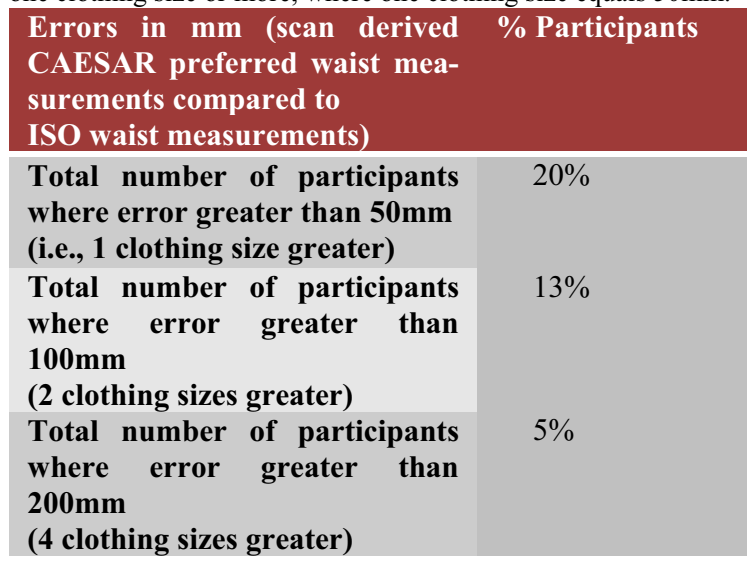

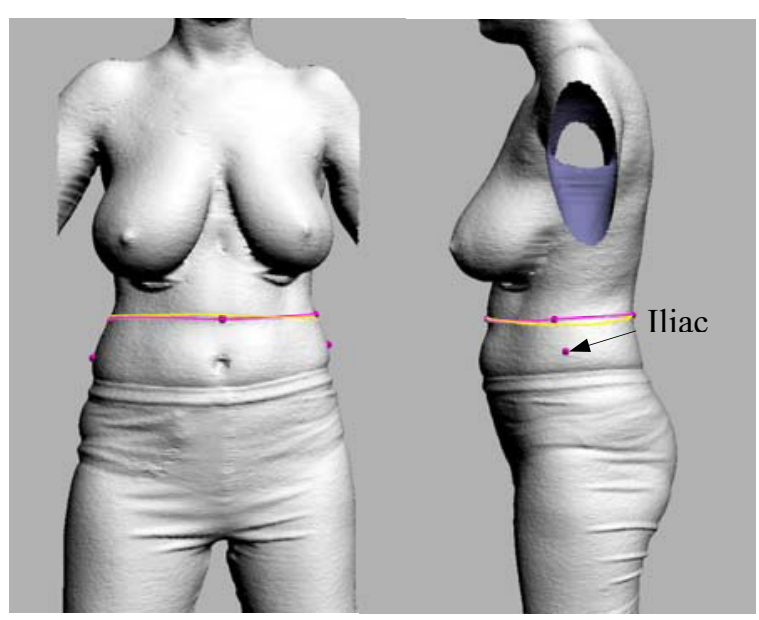

Fig. 3 shows the bodyscan images of B71 front and side view whose ISO and preferred waist definitions are identical. 


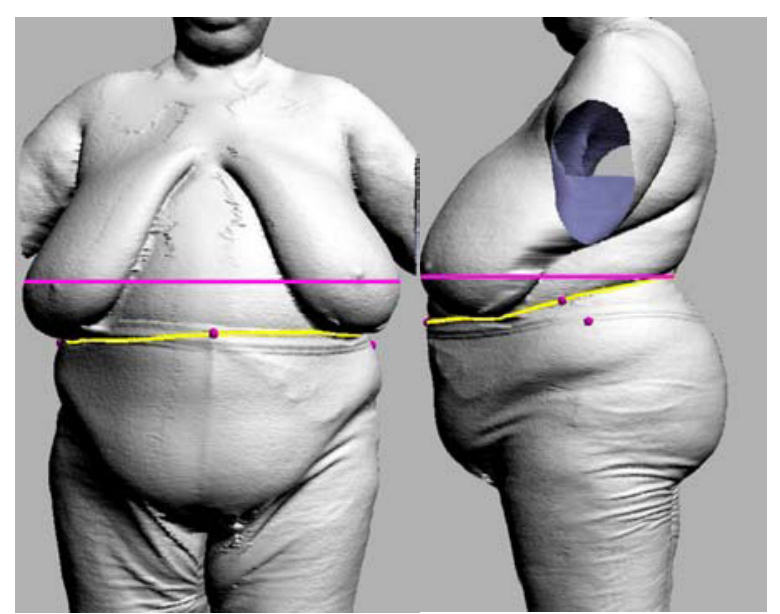

Fig. 4 shows B52 yellow line marking the CAESAR preferred waist and pink line marking the ISO waist.

Table 2 listing measurement of the three subjects shown in Figures 2, 3 and 4

\begin{tabular}{|c|c|c|c|}
\hline $\begin{array}{l}\text { Subject } \\
\text { number }\end{array}$ & $\begin{array}{l}\text { Preferred } \\
\text { waist mm } \\
\quad \text { (Steel } \\
\text { tape } \\
\text { measure) }\end{array}$ & $\begin{array}{l}\text { Preferred } \\
\text { waist mm } \\
\text { (scan } \\
\text { derived) }\end{array}$ & $\begin{array}{c}\text { ISO } \\
\text { waist mm } \\
\text { (scan } \\
\text { derived) }\end{array}$ \\
\hline $\begin{array}{r}\text { B71 } \\
(\text { Fig.3) }\end{array}$ & 741 & 757 & 757 \\
\hline $\begin{array}{r}\text { B52 } \\
(\text { Fig.4) }\end{array}$ & 1278 & 1307 & 1539 \\
\hline $\begin{array}{r}\text { B92 } \\
\text { (Fig.5) }\end{array}$ & 967 & 1078 & 1310 \\
\hline
\end{tabular}

Subject 52, shown in Figure 4, front and side profile has a manual measurement smaller than both scan derived measurements. However the difference in instrument in her case is $29 \mathrm{~mm}$, whereas the difference between measuring the Preferred waist and ISO waist is $232 \mathrm{~mm}$, which is more than 4 clothing sizes; see Table 2 . The validity of this measurement for constructing clothing must be called into question.

Similarly subject B92, shown in Figure 5, has $105 \mathrm{~mm}$ difference between the manually taken tape measure and the scan extracted preferred waist, but a huge $232 \mathrm{~mm}$ difference between the two definitions and $343 \mathrm{~mm}$ (almost seven sizes) difference between the manual tape measure and the scan extracted ISO, see Table 2. In addition, B92 is showing clustering of the bust, waist and hip associated with a short stature; see Figure 5. The scanner measurement for both ISO waist and CAESAR preferred waist is occluded by ptosis of the parenchymal tissue shown in Figure 6. Not only individuals with larger circumference have a problem. Figure 7 shows front, back and side photographs of an individual $165 \mathrm{~cm}$ tall and $53 \mathrm{~kg}$ in weight. When asked to indicate her preferred waist she did so at the level of the lumbar lordosis, which in her case is lower than her iliac crest height and is therefore not within the ISO definition. Her profile picture shows the ISO waist marked with a white line, which if she were to wear pants at that height would create significant discomfort, as the pants would slide down her spine and rest at the lumbar lordosis, creating an unnecessary and unflattering fullness in the crotch length. It appears that the ISO waist definition was formulated when there was insufficient understanding of the range of human anatomical variation that the measurement definition might encounter.

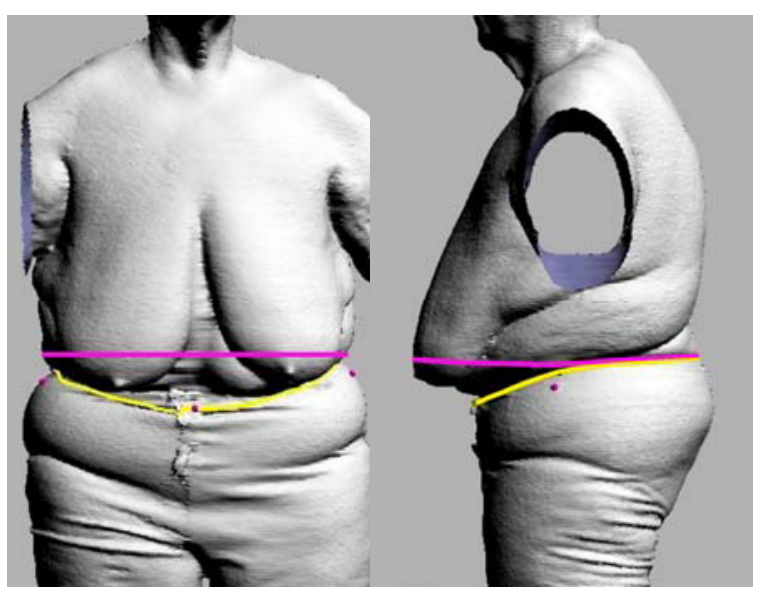

Fig. 5 showing subject B92 with a short trunk clustering vertically the bust, waist and hip. Humans can see the bust is above the waist and hip but the scanner can't. ISO waist is pink and CAESAR waist is yellow.

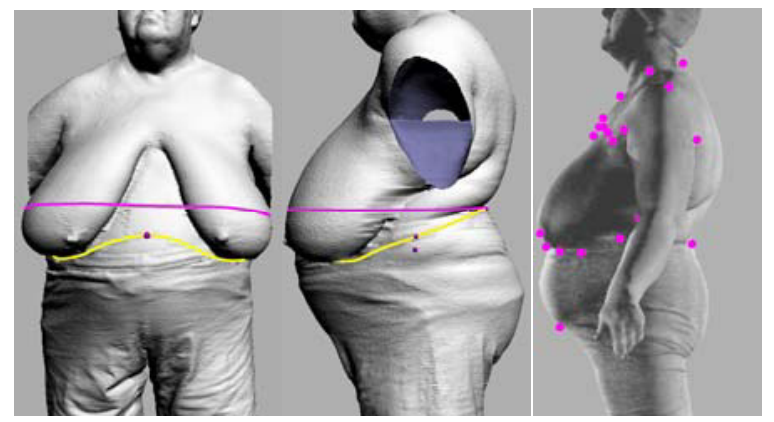

Fig. 6 shows 2 examples of ptosis of the parenchymal tissue (drooping breasts) occluding both scanner measurements of the waist. The manual tape measurement was passed under the breasts. 


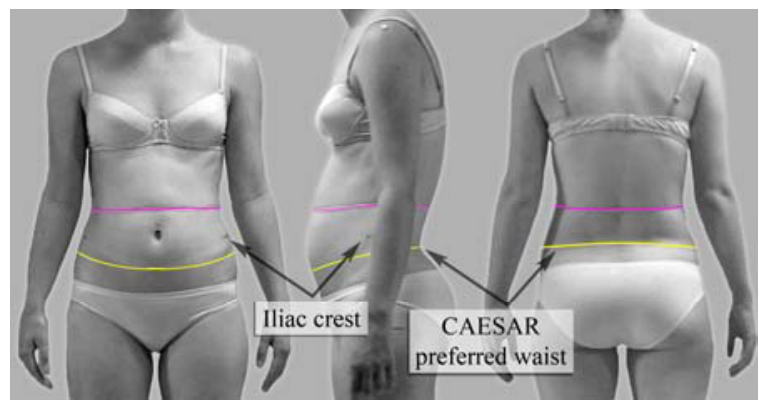

Fig. 7 shows ISO waist marked in pink and preferred waist yellow, an arrow indicates the iliac crest marker. The preferred waist is below the iliac crest. Normally this would be dismissed as a positioning error but here clearly the cause can be seen as an anatomical variation of lumbar lordosis positioned below her iliac crest height. This location discrepancy has implcations for other ISO measurements such as crotch length, body rise etc. which use waist as a landmark.

\section{Discussion}

Although variously defined and measured waist circumferences may serve specific purposes well in various sectors of human activities, the lack of uniformity is disturbing. A waist definition that is not fit-for-purpose, that incorrectly interprets waist definition, or incorrectly measures waist, is likely to result in technical errors that will lead to commercial wastage and customer dissatisfaction.

While the analysis here is focusing on garment construction there are clear implications for incorrect waist measurement in clinical situations that may lead to wrong diagnosis, incorrect treatment and further health problems.

The large discrepancy between the formal ISO waist definition and the "preferred waist" as indicated by participants encourages the conclusion that ISO waist definition is based on an idealized perception of human anatomy. Considerable anatomical variations include substantial abdominal adiposity, position of ilium in relation to lower spine, especially lumbar lordosis, and posture and arrangement of abdominal viscera. Female breasts may have a shape that causes breast tissue to be distributed on or below the waistline in cases of pendulous breasts. Most methods of measurement have been developed for the anatomy of an idealized young adult person of average dimensions within the normal body mass range. However the CAESAR defined preferred waist measurement taken manually with a steel tape was able to measure accurately and validly the waist for garment construction. It could measure underneath pendulous breasts and in fat folds as required, and thereby achieve results similar in position and length to that of a well-fitting waisted garment such as pants or skirt.

Davis reported that the waist must be allowed to slope for correct fit of garments [7]. A study by Veitch et al. shows mean waist tilt (back minus front waist height to ground) of $15 \mathrm{~mm}$ [17] for a population of 1265 adult women [11].

The definition of waist could be improved so that it applied to all humans within the actual, realistic range of anatomical variation and could be uniformly and reliably measured with both traditional and newly developed tools.

Firstly the author suggests the waist be measured manually in two ways. Keep the ISO defined waist but add a second definition to the ISO 8559, based on CAESAR preferred waist. Preferred waist may be horizontal as shown in Figure 2 but it is also allowed to slope as shown in Figures 2, 3 and 4. Therefore ISO would need to add four waist heights, front, back and both sides to replace the existing single waist height (ISO 2.2.3). [3]

There are also 6 measurements in the ISO that use waist as a landmark. These include the following vertical dimensions:

- Waist front length (ISO 2.2.16)

- Center back waist (C7-waist posterior) (ISO 2.2.10)

- Cervical waist anterior (ISO 2.2.14)

and measurements used in pants construction:

- Waist height (ISO 2.2.3)

- Body rise (ISO 2.2.5)

- Crotch length (ISO 2.2.19)

Garment construction uses the preferred waist, therefore these measures are only useful in relation to preferred waist. ISO would need to revise which waist these measures are taken from.

Scanners are an excellent tool if the limitations are known and managed. Without the scan technology large measurement discrepencies could be dismissed as recording and/or transcription error, but with the body scan as a reference it is possible to reexamine the subject to gain an understanding of why the discrepencies occurred and problem solve to improve future work. In addition, the collection of $3 \mathrm{D}$ and $1 \mathrm{D}$ data enabled a breast reduction study to be reanalysed to create a new study for this paper, which when published, shares knowledge on the topic of human variation and measurement.

The limitations are that scan derived measurement for waist circumference is not the same as manual tape measurement and therefore needs to be recognised as a separate measurement. Even when 
controlled for location errors these two instruments cannot be interchanged. ISO needs to indicate the measuring instrument for waist circumference.

The errors in definition and location are not random errors - they are specific to body fat and anatomical variation. So it is not a random group who have problems being measured and the extent to which this group occurs in the wider population is not known. However it is known that if no allowance is made to measure these people they will not be captured in anthropometric surveys and that in turn will preclude their further study. Also it is precisely this group of exceptional individuals that are likely to be test cases in design [15].

In conclusion, a garment survey that uses a $3 \mathrm{D}$ body scanner needs a method inclusive of all the population, i.e., it does not exclude individuals with

\section{References}

[1] ASTM International, D5219-09 Standard Terminology Relating to Body Dimensions for Apparel Sizing, ASTM Standards on Disc, West Conshohocken, PA.

[2] Australian Standard AS1344-1997 Size coding scheme for women's clothing - Underwear, outerwear and foundation garments.

[3] International Organization for Standardization, Garment Construction and Anthropometric Surveys \pm Body Dimensions, Reference No. 8559-1989, International Organization for Standardization, Geneva, 1989.

[4] S. Blackwell, K.M. Robinette, H.A.M. Daanen, M. Boehmer, S. Fleming, S. Kelly, T. Brill, D. Hoeferlin and D. Burnsides, Technical report ARFL-HE-WP-TR-2002-0173, United States Air Force Research Laboratory, Civilian American and European surface anthropometry resource (CAESAR), Final Report 2, Warrendale, PA.

[5] J. E. Brown, J.D. Potter, D.R. Jacobs.Jr, R.A. Kopher, M.J. Rourke, G.M. Barosso, P.J. Hannan and L.A. Schmid, Maternal waist-to-hip ratio as a predictor of newborn size: Results of the Diana Project, Epidemiology 7 (1996), 62-66.

[6] M.J. Chung, H.F. Lin and M.J.J. Wang, The development of sizing systems for Taiwanese elementary- and high-school students, International Journal of Industrial Ergonomics 37 (2007), 707-716.

[7] B. Davis, Towards pattern making technology as a source of competitive advantage, Melbourne, 2000, pp. 9, 60.

[8] P. Dench, CySize software, headus (metamorphosis) www.headus.com.au

[9] A.M. Fullenkamp, K.M. Robinette and H.A.M. Daanen, Gender Differences in NATO Anthropometry and the Implication for Protective Equipment, National Research Council, Soesterberg, The Netherlands, September 2008.

$[10]$ T. Han, E. Van Leer, J. Seidell and M. Lean, Waist circumference action levels in the identification of cardiovascular risk factors: prevalence study in a random sample, British Medical Journal (Clinical research ed.) 311 (1995), 1401-1405.

[11]M. Henneberg and D. Veitch, Australian Size Survey, ISAK Kinanthreport 16 (2003), 34

[12]L. de Koning, A.T. Merchant, J. Pogue and S.S. Anand, Waist circumference and waist-to-hip ratio as predictors of cardi- specific body fat patterns. It is exactly the boundary cases that are used for design, so these are the exact individuals needed as test cases. To be inclusive of the wide range of anatomical variation and provide the most useful future reference tool the survey needs:

- manually placed landmarks,

- a scanner that can recognize landmarks, and

- some manual measurements, in particular, the waist.

The ISO 8559 waist definition needs to be modified to make it more inclusive of the full range of anatomical variation encountered amongst adult women.

ovascular events: meta-regression analysis of prospective studies, European Heart Journal 28 (2007), 850.

[13]M. Kouchi, personal communication about JIS L0111: 1983 confirmed 1992(Glossary of Terms used in Body Measurements for Clothes) corresponds to ISO 3635 (Size designation of clothes - definitions and body measurement procedure). Makiko Kouchi is Prime Senior Researcher, Digital Human Research Center, National Institute of Advanced Industrial Science and Technology. Email to author, 22/08/2011.

[14] G.C. Marks, J.P. Habicht and W.H. Mueller, Reliability, dependability, and precision of anthropometric measurements, American Journal of Epidemiology 130 (1989), 578-587.

[15] K.M. Robinette, and J. Hudson, Chapter 12: Anthropometry, in: Handbook of Human Factors and Ergonomics, $3^{\text {rd }}$ edition, G. Salvendy, ed., John Wiley \& Sons, New York, 2006, pp. 322-339.

[16] K.M. Robinette, and H.A.M. Daanen, Precision of the CAESAR scan-extracted measurements, Applied Ergonomics 37 (2006), 259-265.

[17] D. Veitch, L. Veitch, and M. Henneberg, Sizing for the Clothing Industry Using Principal Component, Analysis-An Australian Example, Journal of ASTM International 4 (2006).

[18] D. Veitch, K. Burford, P. Dench, N. Dean and P. Griffin, Measurement of breast volume using body scan technology (computer-aided anthropometry) publication pending. 